\title{
A broadband leptonic model for gamma-ray emitting microquasars
}

\author{
V. Bosch-Ramon ${ }^{1}$, G. E. Romero ${ }^{2,3, \star}$, and J. M. Paredes ${ }^{1}$ \\ 1 Departament d'Astronomia i Meteorologia, Universitat de Barcelona, Av. Diagonal 647, 08028 Barcelona, Catalonia, Spain \\ e-mail: vbosch@am.ub.es; jmparedes@ub.edu \\ 2 Instituto Argentino de Radioastronomía, C.C.5, (1894) Villa Elisa, Buenos Aires, Argentina \\ e-mail: romero@iar.unlp.edu.ar \\ ${ }^{3}$ Facultad de Ciencias Astronómicas y Geofísicas, UNLP, Paseo del Bosque, 1900 La Plata, Argentina
}

Received 14 June 2005 / Accepted 19 September 2005

\section{ABSTRACT}

Observational and theoretical studies point to microquasars (MQs) as possible counterparts of a significant fraction of the unidentifiedgamma-ray sources detected so far. At present, a proper scenario to explain the emission beyond soft X-rays from these objects is not known, nor what the precise connection is between the radio and the high-energy radiation. We develop a new model where the MQ jet is dynamically dominated by cold protons and radiatively dominated by relativistic leptons. The matter content and power of the jet are both related with the accretion process. The magnetic field is assumed to be close to equipartition, although it is attached to and dominated by the jet matter. For the relativistic particles in the jet, their maximum energy depends on both the acceleration efficiency and the energy losses. The model takes into account the interaction of the relativistic jet particles with the magnetic field and all the photon and matter fields. Such interaction produces significant amounts of radiation from radio to very high energies through synchrotron, relativistic Bremsstrahlung, and inverse Compton (IC) processes. Variability of the emission produced by changes in the accretion process (e.g. via orbital eccentricity) is also expected. The effects of the gamma-ray absorption by the external photon fields on the gamma-ray spectrum have been taken into account, revealing clear spectral features that might be observed. This model is consistent to the accretion scenario, energy conservation laws, and current observational knowledge, and can provide deeper physical information of the source when tested against multiwavelength data.

Key words. X-rays: binaries - stars: winds, outflows - gamma-rays: observations - gamma-rays: theory

\section{Introduction}

Microquasars are X-ray binary systems (XRBs) with relativistic bipolar outflows or jets (Mirabel \& Rodríguez 1999). These extended structures have been observed in galactic objects at radio wavelengths from the seventies (SS 433; Spencer 1979; Hjellming \& Johnston 1981). The inner region of the disk emits thermally at soft X-rays, losing accretion kinetic energy through viscosity-related phenomena. Additionally, there seems to be evidence supporting the existence of a hot relativistic plasma around the compact object (called "corona" hereafter), which is radiatively significant during the so-called lowhard state of the source. Corona electrons would Comptonize soft disk photons to higher energies (McClintock \& Remillard 2004). Transport of angular momentum and kinetic energy linked to a specific inner disk magnetic field configuration could lead to the formation of a jet (Meier 2003).

The detection of extended non-thermal radio emission provided clear evidence for the presence of relativistic leptons

* Member of CONICET. in the jets of MQs, although it was not considered in general that jets could emit significantly at X-rays or beyond. Paredes et al. (2000) proposed the microquasar LS 5039 as the counterpart of the EGRET source 3EG J1824-1514 (Hartman et al. 1999). In their scenario, the jet relativistic electrons scatter the photons of the massive stellar companion, showing that microquasar jets are possible sources of gamma-rays. Further statistical and theoretical studies showed that microquasars could be behind some of the unidentified gamma-ray sources in the Galaxy (Kaufman Bernadó et al. 2002; Romero et al. 2004; Bosch-Ramon et al. 2005a,b). Observational evidence of jets as high energy emitters came from the detection of X-ray extended emission (e.g., in SS 433, Marshall et al. 2002; Migliari et al. 2002; XTE J1550-564, Corbel et al. 2002; and $\mathrm{H}$ 1743-322, Corbel et al. 2005). The recent detection by the ground-based Cherenkov telescope HESS of TeV emission coming from the microquasar LS 5039 (Aharonian et al. 2005) largely confirms the association proposed by Paredes et al. (2000), and it is strong evidence that microquasars are sources of very high-energy gamma-rays, their jets being the best candidates to generate such emission. 
For the modeling of gamma-ray emission from jets of MQs, there have been two types of approach. One considers that hadrons lead radiative processes at $\mathrm{GeV}-\mathrm{TeV}$ gamma-rays and beyond (Romero et al. 2003, 2005; Romero \& Orellana 2005), producing detectable amounts of neutrinos (Torres et al. 2004), and leaving electrons as possible significant emitters only at lower energies. These are the so-called hadronic models. The other approach extends the energy of leptons from synchrotron radio emitting energies exploring inverse Compton and/or synchrotron emission in the jets (i.e. Atoyan \& Aharonian 1999; Markoff et al. 2001; Georganopoulos et al. 2002; Bosch-Ramon et al. 2005a). These are the so-called leptonic models. All these models developed so far are important to investigate to what extent each mechanism of emission would be relevant under different circumstances, and what level of physical detail is required for realistic modeling with the available observational data. Nevertheless, a comprehensive MQ jet model attempting to explain emission properties in the whole range of spectral frequencies, in accordance with the energy and matter constraints imposed by accretion and the conservation energy law at the microscopic level, is still lacking.

In this paper, we investigate persistent MQ compact jets to give multiwavelength and variability predictions consistent with the MQ scenario as a whole. We use the term "compact jet" or "jet" referring to the type of outflows thought to be present during the low-hard state (Fender et al. 2003a). The extended radio lobes, which are also observed in some MQs (e.g. 1E 1740.7-2942; Mirabel et al. 1992), and the blobs ejected during state transitions (e.g. GRS 1915+105, Mirabel et al. 1998) are not considered as compact jets. "Consistent" means here to develop the model taking into account the total amount of matter available for accretion, the pressure relationship between compact jets and their environments, the standard models for accretion and jet ejection, the mechanism for particle acceleration, the pair creation and annihilation rates and the law of microscopic energy conservation. Semi-analytical calculations have been implemented to compute all the significant emission and absorption mechanisms that take place in the jet: synchrotron, relativistic Bremsstrahlung with internal and external matter fields, inverse Compton with internal and external photon fields, and creation and annihilation of pairs. In a forthcoming paper, we will present an application of the present model to the microquasar LS 5039 (Paredes et al. 2005).

In Sect. 2, the general picture of the model is presented; in Sect. 3, the details of the jet model are given; in Sect. 4, the considered radiative processes are explained. The resulting spectral energy distributions (SEDs) for several relevant situations are shown and discussed in Sect. 5, as are the variability properties of the model. Other questions implied by our model are discussed in Sect. 6, and all the treated issues are summarized in Sect. 7.

\section{General picture}

The MQ scenario considered here consists of a binary system formed by a star, either of low or high mass, and a compact object, either a black hole or a neutron star. At this stage, the nature of the compact object is not relevant. The stellar companion feeds the accretion process onto the compact object. Part of the energy associated with the accreted matter is radiated in an accretion disk, and part is converted to kinetic and magnetic energy of the accretion flow under the effects of the compact object potential well. In the low-hard state, when the accretion rate is moderately low, the accretion disk is well described by a standard optically thick and geometrically thin Keplerian disk (Shakura \& Sunyaev 1973) up to a certain transition radius, assumed here to be $R_{\text {disk }} \sim 50 R_{\text {Sch. }}$. At distances to the compact object smaller than the transition radius, we assume the existence of a hot plasma (the corona), whose relativistic electrons scatter soft disk photons from the geometrically thin disk. The properties of this inner region are considered to be suitable for jet ejection phenomena to take place (Meier 2003). The energetics of the jet is assumed to be dominated by accretion, and further energy sources like compact object rotation have been neglected (an approximation that seems to be reasonable, see Hujeirat 2004). A more extended discussion on jet formation and energetics is present in Sect. 3.1.

If the jet is formed by accreted matter, it will contain protons and electrons, as well as a magnetic field $(B)$ associated with the plasma. Our assumption is that the matter kinetic luminosity is higher than the magnetic luminosity (or total magnetic energy crossing a jet cross-section per time unit) in the jet regions we are concerned with, although the magnetic field can be still significant once the jet is formed, since the ejection mechanism is likely to be magneto-hydrodynamical. For simplicity, the jet is supposed to be perpendicular to the orbital plane. An important fact is that variability can be easily reproduced when the accretion rate is not constant due to, e.g., orbital eccentricity. If protons are relativistic and a confining mechanism is absent, a mildly relativistic jet (see Sect. 3.2) will expand relativistically, appearing not collimated. Therefore, cold protons instead of relativistic protons should dominate the jet pressure, with free expansion speeds smaller than in a full relativistic jet, allowing for a small jet opening angle. The shock acceleration mechanism, assumed at this stage to take place all along the jet due to velocity variations in the ejected matter (Rees 1978), can effectively accelerate particles, but must such particles diffuse through the shock. This condition is fulfilled only by a small fraction of protons that, assuming they follow a thermal distribution, populate the highest energy tail. The condition that particles must diffuse through the shock to be effectively accelerated imposes a more restrictive condition on the electrons since, in general, they have a diffusion mean free path much smaller than protons (i.e. lower temperatures), it being unlikely that shock acceleration will operate significantly on any fraction of the electron thermal distribution that also forms the jet. Therefore, we assume that an unspecified injection mechanism, like some kind of plasma instability, operates on the jet electrons heating them enough so they diffuse through the shock (see Sect. 3.2).

In this scenario, radiative processes that take place in the jet can produce significant emission in the whole spectrum (see Sects. 4 and 5). Although there could still be enough protons to be significant from the radiative point of view, treatments on them can be found elsewhere (Romero et al. 2003, 2005), 


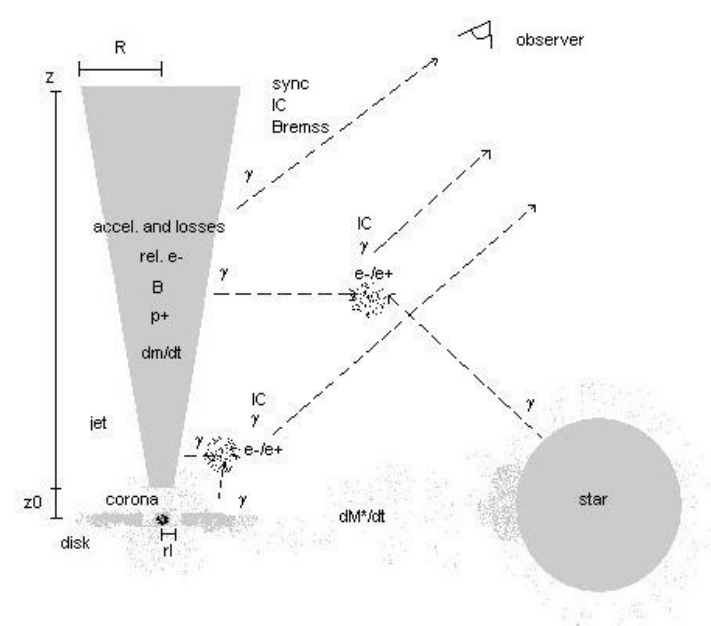

Fig. 1. Picture of the microquasar scenario. The main features considered by the model are listed in their associated region: accretion and jet matter rate, magnetic field, particle acceleration, energy losses, leptonic emission, pair creation and secondary emission. The plot is not to scale.

and we will focus on the leptonic component only ${ }^{1}$. Synchrotron emission can be important from radio to soft gamma-rays, if the magnetic field is close to equipartition with matter. Moreover, in the regions close to the jet ejection point, magnetic and relativistic particle densities are high enough for synchrotron self-Compton process (SSC) to be considered. Moreover, other self-Compton processes can occur, although SSC will be dominant in most cases. IC interactions between jet electrons and external photon fields (external Compton or EC) can also be significant. If the companion star is massive, stellar IC scattering can be dominant at the highest energies. EC scattering with disk and corona photon fields could be non-negligible as well, and relevant for a low-mass system (see, nonetheless, Grenier et al. 2005). All IC fields appear to dominate emission from soft gamma-rays to very high-energy gamma-rays (VHE), although a relativistic Bremsstrahlung component can also reach very high energies, generally being a minor component. Besides radiative processes, absorption processes are to be taken into account regarding gamma-ray radiation created in the jet. Absorption of photons by pair creation can be significant at the base of the jet, and also significant within the binary system if a massive and very luminous star is present. Moreover, secondaries created by electromagnetic cascades should be considered, since their contribution is not negligible. In this work, we provide a rough estimate of these effects on the observed spectrum. It is discussed in Sects. 4 and 5. For clarity, in Fig. 1, we show a rough sketch of the microquasar scenario.

Four different cases are explored in this paper. First, a high-mass and a low-mass XRB presenting a jet with a high particle acceleration efficiency are studied in case A and B,

1 We note that the total luminosity radiated by relativistic protons through proton-proton interaction should be close to that calculated for relativistic Bremsstrahlung due to similar cross-sections and target densities, assuming the same energy distribution for both relativistic protons and relativistic electrons. respectively. For these two particular scenarios, the accretion disk and the corona have been taken to be faint. Here, the effect of the star on the predicted spectra is investigated. In case $\mathrm{C}$, the model is applied to a high-mass system considering luminous accretion disk and corona with a low particle acceleration efficiency. For these three cases the velocity is fixed to a mildly relativistic value. This provides restrictions to the fraction of accreted matter that is ejected, since the energy budget is limited and some part is radiated during the accretion process. Also, the different acceleration efficiency has a strong effect on the spectrum at the higher energies. In case $\mathrm{D}$, a light jet pointing towards the observer without a speed restriction and with a faint disk and corona is investigated. A high particle acceleration efficiency has also been considered for this particular scenario, which shows that the jet could be a very strong emitter of X-rays and gamma-rays under suitable conditions, appearing almost as an ultra luminous X-ray source (ULXs) if located at large distances. We recall that it is supposed that all these systems are in a low-hard-like state, when compact jets are thought to be present with rather stable characteristics (e.g. Gallo et al. 2003).

\section{The model: formation and properties of the jet}

We describe in this section the simple scenario we adopt concerning the formation, collimation and other properties of the jet but the radiative ones, which are given in Sect. 4 . The fixed parameters of the model, their representative symbols, description and values are provided in Table 1, being discussed in the text. For the stellar mass loss rate $\left(\dot{m}_{\mathrm{w}}\right)$, the jet size, and the orbital parameters, we have used typical MQ values. The parameter values that vary for the four specific scenarios A-D are given below the fixed ones in Table 1.

\subsection{Jet formation}

\subsubsection{Stellar matter accretion}

The accretion rate adopted in this work has been estimated assuming that the system is accreting at $5 \%$ of the Eddington value, thought to be typical for accreting XRBs in the low-hard state (Esin et al. 1997). For the accretion luminosity to accretion rate ratio, we have adopted $0.05 c^{2}$, which for a compact object of $3 M_{\odot}$ yields an accretion rate of $6 \times 10^{-9} M_{\odot} \mathrm{yr}^{-1}$. Assuming a spherical Bondi-Hoyle accretion model (Bondi 1952, see also Reig et al. 2003), in the case of high-mass MQs, with typical stellar mass loss rates of about $10^{-6} M_{\odot} \mathrm{yr}^{-1}$ and high wind velocities of few times $10^{8} \mathrm{~cm} \mathrm{~s}^{-1}$ inferred from spectroscopic observations, the mentioned moderate accretion rate is hard to achieve. It likely implies that some anisotropy in the wind properties occurs in the direction towards the compact object. We have assumed a low wind velocity in the direction of the compact object that can be estimated from the stellar mass loss and accretion rates quoted above (see also Table 1), obtaining values of $\lesssim 10^{8} \mathrm{~cm} \mathrm{~s}^{-1}$. Moreover, the adoption of an accretion model allows us to provide a rough estimate of the effects of eccentric orbital motion on emission variability as presented in Sect. 5, normalizing the accretion rate to the quoted value at phase 0 . We adopt the convention here that phase 0 is the 
Table 1. Model parameters.

\begin{tabular}{|c|c|c|c|c|}
\hline Parameter: description [units] & Values & & & \\
\hline$e$ : eccentricity & 0.3 & & & \\
\hline$a$ : orbital semi-major axis $\left[R_{\odot}\right]$ & 45 & & & \\
\hline$\dot{m}_{\mathrm{w}}:$ stellar mass loss rate $\left[M_{\odot} \mathrm{yr}^{-1}\right]$ & $10^{-6}$ & & & \\
\hline$k T_{\text {disk }}:$ disk inner part temperature $[\mathrm{keV}]$ & 0.1 & & & \\
\hline$p_{\text {cor }}:$ corona photon index & 1.6 & & & \\
\hline$R_{\text {disk }}:$ disk inner radius $\left[R_{\mathrm{Sch}}\right]$ & 50 & & & \\
\hline$r_{1}$ : launching radius $\left[R_{\mathrm{Sch}}\right]$ & 4 & & & \\
\hline$z_{0}:$ jet initial point in the compact object $\mathrm{RF}\left[R_{\mathrm{Sch}}\right]$ & 50 & & & \\
\hline$\chi:$ jet semi-opening angle tangent & 0.1 & & & \\
\hline$\varrho:$ equipartition parameter & 0.1 & & & \\
\hline$\zeta:$ max. ratio hot to cold lepton number & 0.001 & & & \\
\hline$q_{\text {acc }}$ : fraction of the Edington accretion rate & 0.05 & & & \\
\hline \multirow[t]{2}{*}{$p$ : electron power-law index } & 2.2 & & & \\
\hline & $\mathrm{A}$ & $\mathrm{B}$ & $\mathrm{C}$ & $\mathrm{D}$ \\
\hline$M_{\mathrm{x}}:$ compact object mass $\left[M_{\odot}\right]$ & 3 & 3 & 3 & 15 \\
\hline$R_{\star}:$ stellar radius $\left[R_{\odot}\right]$ & 15 & 1 & 10 & 10 \\
\hline$M_{\star}:$ stellar mass $\left[M_{\odot}\right]$ & 30 & 1 & 20 & 20 \\
\hline$L_{\star}:$ stellar bolometric luminosity [ $\mathrm{erg} \mathrm{s}^{-1}$ ] & $10^{39}$ & $10^{33}$ & $10^{38}$ & $10^{38}$ \\
\hline$T_{\star}:$ stellar surface temperature $[\mathrm{K}]$ & $4 \times 10^{4}$ & $6 \times 10^{3}$ & $3 \times 10^{4}$ & $3 \times 10^{4}$ \\
\hline$\kappa:$ jet-accretion rate parameter & 0.05 & 0.05 & 0.02 & 0.01 \\
\hline$\xi:$ shock energy dissipation efficiency & 0.25 & 0.25 & 0.05 & 0.5 \\
\hline$\theta$ : jet viewing angle $\left[{ }^{\circ}\right]$ & 45 & 45 & 45 & 1 \\
\hline$\eta$ : acceleration efficiency & 0.1 & 0.1 & 0.0001 & 0.1 \\
\hline$\alpha_{\text {disk }}:$ disk radiative efficiency & 0.025 & 0.025 & 0.25 & 0.025 \\
\hline$\alpha_{\text {cor }}:$ corona radiative efficiency & 0.005 & 0.005 & 0.05 & 0.005 \\
\hline
\end{tabular}

periastron passage and when the compact object is in opposition to the observer. We note that periastron passage does not correspond to the accretion peak, due to effects concerning the composition of velocities for both the compact object and the wind. In the case of low-mass MQs, orbital variability is not explored here because these systems present circular orbits.

\subsubsection{Ejection velocity of the jet}

The jet velocity is estimated taking into account the amount of available kinetic luminosity that can be extracted from the accretion at the launching radius $r_{1}$ (see Table 1 and Sect. 6). $r_{1}$ is not the distance at which the jet is formed, but a characteristic radius where ejected matter gets extra kinetic energy from the accretion reservoir. The knowledge of such a quantity could help us to understand where ejection originates, although ejection is likely to be a spatially extended phenomenon. It is necessary to establish the matter rates of both the advected and the ejected matter components that, in relation to the total accretion rate, follow the formula:

$\dot{m}_{\mathrm{acc}}=2 \dot{m}_{\mathrm{jet}}+\dot{m}_{\mathrm{adv}}$.

The factor 2 is due to the existence of a jet and its counterjet. We introduce the parameter $\kappa$ through the relationship: $\dot{m}_{\text {jet }}=$ $\kappa \dot{m}_{\text {acc }}$. To assign a certain amount of extra kinetic luminosity to the ejected matter, we have taken into account the energy dissipated in the disk and the corona in the form of radiation, as well as the energy borne by the advected matter after transferring part of its kinetic energy to the jet. This remaining advected kinetic luminosity has been assumed to be associated with the Keplerian velocity at the launching radius, which is:

$$
L_{\mathrm{k} \text { adv }}\left(r_{1}\right) \sim \frac{R_{\mathrm{Sch}}}{4 r_{1}} \dot{m}_{\mathrm{adv}} c^{2} .
$$

The previous considerations give a first order estimate of the injection jet velocity. The equation we obtain is:

$L_{\mathrm{acc}}\left(r_{1}\right)=L_{\mathrm{k} \text { jet }}+L_{\mathrm{k} \mathrm{adv}}+L_{\mathrm{disk}}+L_{\mathrm{cor}}$.

$L_{\mathrm{k} \text { jet }}$ is the jet kinetic luminosity at the launching radius. This accounts for the kinetic luminosity required to carry the jet matter outside the potential well (e.g. it could be in the form of magnetic luminosity in those regions, before the jet is completely formed at the assumed distance of $50 R_{\text {Sch }}$ ) plus the kinetic luminosity of the jet after ejection:

$L_{\mathrm{k} \text { jet }}\left(r_{\mathrm{l}}\right)=\frac{G M_{\star} 2 \dot{m}_{\mathrm{jet}}}{r_{\mathrm{l}}}+\left(\Gamma_{\mathrm{jet}}-1\right)\left(2 \dot{m}_{\mathrm{jet}}\right) c^{2}$.

$\left(\Gamma_{\text {jet }}-1\right)\left(2 \dot{m}_{\text {jet }}\right) c^{2}$, the jet matter kinetic luminosity, accounts also for the magnetic field and relativistic particle luminosity 
since, once formed, the jet is assumed to be cold matter dominated. $L_{\mathrm{disk}}$ is the disk radiated luminosity, taken to be a few $\%$ of $L_{\mathrm{acc}}$, and $L_{\mathrm{cor}}$ is the corona radiated luminosity, about $1 \%$ or less of $L_{\text {acc }}$ (the efficiencies that we have adopted are similar to those found in the literature; McClintock \& Remillard 2004): $L_{\text {disk }}=\alpha_{\text {disk }} L_{\text {acc }}$ and $L_{\text {cor }}=\alpha_{\text {cor }} L_{\text {acc }}$. The final expression for the $\Gamma_{\text {jet }}$ is:

$\Gamma_{\text {jet }}\left(r_{1}\right)=1+\frac{1}{2 \dot{m}_{\text {jet }}}\left(\frac{R_{\text {Sch }}}{4 r_{1}} \dot{m}_{\text {adv }}-\frac{L_{\text {disk }}+L_{\text {cor }}}{c^{2}}\right)$.

We note that the energy dissipated in the shocks formed in the jet is extracted from the corresponding jet kinetic luminosity after ejection, and the velocity at infinity has an associated kinetic luminosity such that $L_{\mathrm{k} \infty}<L_{\mathrm{k} \text { esc }}$. Therefore, the ejection jet Lorentz factor will be larger than its observable value, $\Gamma_{\infty}$, although both will be related by the amount of energy dissipated in the jet (see below). This approach is classical, just to provide a zeroth order estimate.

In Sect. 5 the computed SEDs for four different cases are presented. Except in the case of D, $\Gamma_{\text {jet }}$ is fixed to 1.5. This will allow us to compare, through Eq. (5), the amount of matter carried by the jet between the case when disk/corona emission is weak and the case when it is strong. This will imply that, for the same ejection velocity, the jet in the former case can be heavier than in the latter one (see the corresponding $\kappa$ values in Table 1).

\subsubsection{Magnetic field}

Although we do not consider any particular theory of jet ejection, it is supposed that the mechanism is a magnetohydrodynamic one. If the jet is ejected by converting magnetic energy to matter kinetic energy, it seems likely that $B$ close to the compact object must be beyond equipartition with jet matter. We assume that $B$ goes down as it transfers energy to the jet matter, accelerating it. Finally, $B$ becomes dynamically dominated by jet matter (in the jet reference frame $(\mathrm{RF})$ ). It is assumed that, when the jet is already formed, the magnetic field is entangled with matter and approximately turbulent. Both matter and $B$ follow adiabatic evolution when moving along the jet, with the energy density $\propto 1 / z^{2}$ for a conical jet, where $z$ is the distance to the compact object.

The transition from a magnetic field dominated jet to a matter dominated jet should not be discontinuous; $B$ must reach values below equipartition in relation to matter, but not by too much, since their energy densities should evolve in a similar way once the former is attached to the latter. A more extended discussion of this issue concerning extragalactic jets can be found in Sikora et al. (2005). In our work, the magnetic field in the jet reference frame at different distances from the compact object has been calculated as follows:

$B(z)=\sqrt{\varrho 8 \pi e_{\mathrm{p}}}$,

where, for a cold proton dominated jet, the jet matter energy density is:

$e_{\mathrm{p}}(z)=\frac{\dot{m}_{\text {jet }}}{\pi R_{\text {jet }}^{2} V_{\text {jet }} m_{\mathrm{p}}}\left\langle E_{\mathrm{p} \mathrm{k}}\right\rangle=\frac{\dot{m}_{\text {jet }}}{2 \pi z^{2}} V_{\text {jet }}$.

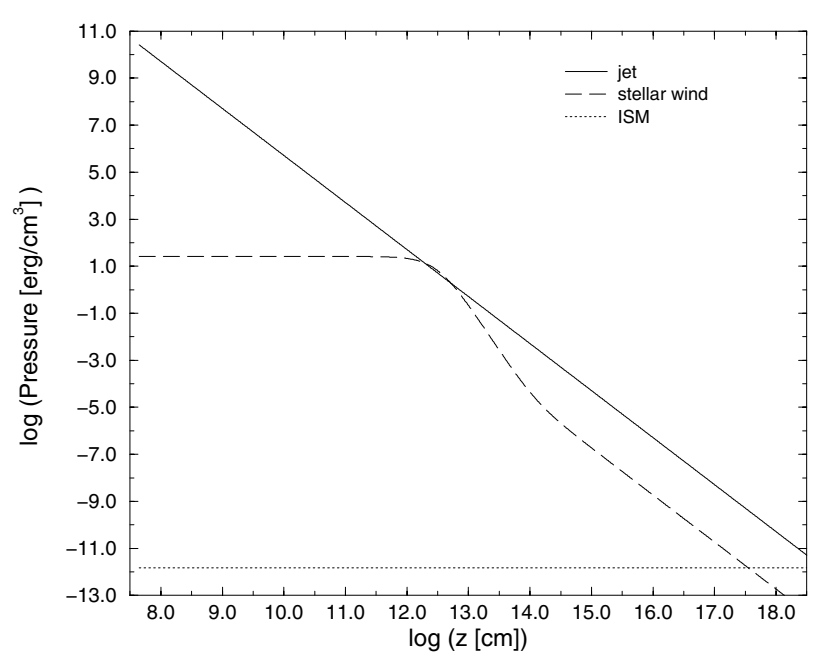

Fig. 2. Pressure values along the jet for the jet matter (solid line), stellar wind (dashed line) and the ISM (dotted line). To compute this, $\kappa=0.05$ and a ISM with a density of $10 \mathrm{~cm}^{-3}$ and a temperature of $10^{3} \mathrm{~K}$ have been adopted.

We introduce $R_{\text {jet }}=\chi z$, and $\left\langle E_{\mathrm{p} \mathrm{k}}\right\rangle$, which is the mean cold proton kinetic energy, taken to be the classical kinetic energy of protons with velocity equal to the expansion velocity $\left(V_{\exp }=\chi V_{\text {jet }}\right)$. This would correspond approximately to the sound speed of the plasma in the jet RF.

\subsection{Jet properties}

\subsubsection{Confinement}

Compact jets in microquasars appear to be in general mildly relativistic (for LS 5039, see Paredes et al. 2002; for LS I +61 303, see Massi et al. 2004; in general, see Gallo et al. 2003). This implies that these jets cannot be collimated by relativistic motion, and an external or internal factor must collimate them. External medium collimation operates when its pressure is similar to or larger than the jet lateral pressure, both taken as a non relativistic ideal gas (the jet expansion velocity considered here, as well as the environment gas, is not relativistic). Environment pressure can be exerted by expelled stellar matter (e.g. stellar wind) and the interstellar medium (ISM). In Fig. 2, we show the jet pressure along the jet, the stellar wind pressure and the ISM pressure. The jet specific parameter values relevant here are those corresponding to the case A. For the ISM, we have adopted a density of $10 \mathrm{~cm}^{-3}$ and a temperature of $10^{3} \mathrm{~K}$, assuming that it is heated by the massive star. For this particular case, the wind velocity has been fixed to $10^{8} \mathrm{~cm} \mathrm{~s}^{-1}$. As is seen in Fig. 2, the pressure of the wind is not significantly above the jet pressure anywhere along the jet. There is a clear change of behavior of the wind pressure on the jet when it turns from kinetic to thermal pressure, at distances similar to the binary system size. We note that ISM effects could be nonnegligible at very large scales. We have not studied the interaction of the jet with the ISM, although a treatment of this kind can be found elsewhere, e.g., in Heinz (2002). Further sources of external influence could be disk radiation and/or disk winds. However, the former does not appear to be effective at middle 
and large jet scales (see, e.g. Fukue et al. 2001), and the latter, if effective beyond disk scale distances, would need to be as compact as the jet itself, and the problem of collimation is transferred to this compact disk wind.

Internal collimation could be achieved by a special magnetic field configuration. Nevertheless, since the magnetic field energy density is considered to be lower than the matter energy density (see Sect. 3.1), this mechanism does not seem to be relevant here. In our context, the jet expands freely at roughly its sound speed, and this type of jet can only be collimated if the plasma sound speed is low, implying a cold matter dominated jet. For instance, the jet of SS 433 is particularly heavy among MQ compact jets and seems to be cold matter dominated, since there is significant thermal $\mathrm{X}$-ray radiation coming from the jet (see, i.e., Fender et al. 2003b). Jet opening angles are typically of a few degrees (for LS 5039, see Paredes et al. 2002; for SS 433, see Marshall et al. 2002). This means that the parameter $\chi$ must be similar to or less than 0.1 (see Table 1). For mildly relativistic jets, if their cold components are in thermal equilibrium, the temperature associated with $V_{\exp }$ will be similar to that of the inner regions of the accretion disk or corona (several $100 \mathrm{keV}$ ).

In order to obtain some upper limits for the amount of accelerated particles, we have computed both the cold and the hot proton pressure along the jet. For hot particles, we have estimated their pressure as corresponding to a relativistic ideal gas of protons following a power-law distribution. It is found that the pressure of the hot component is similar to that of the cold component for a ratio of hot particle to cold particle number of about $1 / 1000$. We have adopted this value for $\zeta$, the parameter that gives the maximum possible ratio of hot to cold lepton number (we assume the existence of one cold lepton per cold proton). We note that the energy density of relativistic leptons is below the magnetic one all along the jet. This fact could imply a better confinement for such particles, considering that the gyro-radius becomes smaller as the magnetic field increases.

\subsubsection{Relativistic particles}

Internal shocks due to different velocities of the jet plasma (Rees 1978; Spada et al. 2001; Yuan et al. 2005) can dissipate bulk kinetic energy, converting it into random kinetic energy of accelerated particles. The injected particle spectrum is kept all along the jet up to a certain maximum energy, which varies in accordance with the balance of energy gains and losses. In addition, the assumption that the jet is cold matter dominated stringently constrains $\zeta$ to the quoted value. Effects of cooling on the power-law spectrum cannot be considered at this stage due to the uncertainties concerning the injection/acceleration processes, and its shape index is therefore fixed. Accelerated leptons emitting synchrotron radiation can produce jet emission similar to that observed at radio wavelengths in LS 5039 (Paredes et al. 2000), the microquasar detected by HESS.

Under the conditions assumed here, the first order Fermi mechanism cannot accelerate thermal electrons (Bell 1978) efficiently. Therefore, a still unspecified mechanism that can accelerate thermal electrons must operate up to the minimum
Fermi acceleration energy, $\gamma_{\min }$. To determine $\gamma_{\min }$, we have assumed that the accelerated leptons have a mean free path within the shock similar to the mean free path of the particles that form the shock, i.e. the jet cold protons. The injection mechanism remains unspecified (e.g. magnetic reconnection that heats electrons, some type of proton-electron temperature coupling, injection of relativistic pairs, etc.), and the injection rate will depend on acceleration efficiency constraints. The Fermi mechanism provides the energy of radiating leptons, and not this unknown injection mechanism. Thus, the radiative properties do not rely on a mysterious process of particle injection but on the well-known first order Fermi theory of particle acceleration (a similar approach to hadron acceleration is performed in Mastichiadis \& Kirk 1995).

The relativistic lepton energy distribution in the RF of the jet is assumed to follow a power-law:

$n_{\mathrm{e}}(\gamma, z)=\frac{N_{\mathrm{inj}}}{\pi(\chi z)^{2} V_{\text {jet }}} \gamma^{-p}$

where $N_{\text {inj }}$ is the injection normalization parameter for the energy distribution of relativistic leptons, which is taken to vary as a function of the acceleration mechanism efficiency and jet conditions (see below). The injected lepton rate $\dot{Q}_{\text {inj }}$ (i.e. the total number of leptons crossing a jet section at $z$ per second) is associated with $N_{\mathrm{inj}}$, and can be obtained from the latter integrating $N_{\text {inj }} \gamma^{-p}$ from $\gamma_{\min }$ to $\gamma_{\max }$. $p$ is taken to be 2.2, a reasonable value that can be derived from optically thin radio spectra observed in some MQs. Since the matter density decays as $1 / z^{2}$ in a conical jet, we observe the $z$-dependence in Eq. (8). The acceleration process is assumed to keep the same energy distribution for the relativistic leptons along the jet, although the maximum energy of the accelerated particles depends on energy loss conditions and the size of the accelerator, taken here to be the jet width. We will neglect at this stage the effects of escaping particles on the particle energy distribution (for a detailed treatment on this see Atoyan \& Aharonian 1999). The power-law is simply cut at a certain $\gamma_{\max }$, which is computed as explained below. Nevertheless, since the accelerator/jet size limits the acceleration efficiency, escape losses are taken into account as cooling terms. We note that a low energy particle population could be present in the jet just below the acceleration injection energy, that can have Lorentz factors $\lesssim 100$. These particles cannot radiate significantly, due to their long radiative timescales and because of adiabatic losses, at least while they are confined within the jet.

\subsubsection{Particle acceleration and jet deceleration}

We have adopted Fermi first order acceleration theory to calculate the maximum energy of accelerated particles in the jet (see, e.g., Biermann \& Sttritmatter 1987; Protheroe 1999). We set the acceleration rate equal to the rate at which particles lose their energy. Expressed in terms of the Lorentz factor:

$\dot{\gamma}_{\text {gain }}=\dot{\gamma}_{\text {loss }}$.

The energy gain rate can be calculated from:

$\dot{\gamma}_{\text {gain }}=\frac{\eta q_{\mathrm{e}} B c}{m_{\mathrm{e}} c^{2}}$, 
where $\eta$ can have different values depending on the shock conditions ${ }^{2}$. For simplicity and since the specific shock conditions are not known, $\eta$ is treated here as a free parameter in the range $10^{-4}-10^{-1} \cdot \eta$ is likely a function of $z$, although at this stage is set to be constant. We recall that $B$ goes down by $1 / z$. The energy loss rate can be estimated adding the contribution of the different types of energy losses. On the one hand, there are adiabatic losses:

$\dot{\gamma}_{\text {adiab }}=\frac{2 V_{\text {exp }} \gamma}{3 R_{\text {jet }}}$,

since relativistic leptons are exerting work against the jet confining walls, whose expansion is led by cold protons. On the other hand, there are radiative losses due to synchrotron, IC and relativistic Bremsstrahlung processes. Hence, we obtain:

$\dot{\gamma}_{\text {loss }}=\dot{\gamma}_{\text {rad }}+\dot{\gamma}_{\text {adiab }}$

The expressions for the particle energy loss rates for each radiative mechanism can be found, e.g., in Blumenthal \& Gould (1970). From these expressions together with Eq. (9), one can finally obtain $\gamma_{\max }$ and its evolution with $z$.

There is a limit for the total amount of energy available for shock acceleration, which is likely related to the shock efficiency to dissipate energy via heating jet leptons. The upper limit for shock energy dissipation efficiency is that $100 \%$ of the kinetic energy is dissipated. It seems unlikely that a so efficient process takes place but, still, an important amount of the energy is released in the form of radiation, as it appears to happen for extragalactic jets and some galactic jets. We have adopted the criterion that, at most, the shock could dissipate up to some fraction of the flow average kinetic energy accelerating particles in the whole jet, or $\xi=0.05,0.25,0.5$ (see Table 1). It is not stated that a 5,25 or $50 \%$ of the whole jet kinetic energy goes to the relativistic particles. The shock could dissipate enough energy to accelerate the maximum number of relativistic leptons in the jet (given by $\zeta$ ), still being below the efficiency limit. This implies that for most of the jet the energy dissipation rate can be below this constraint. If a large fraction of the jet kinetic energy goes to heat particles, the shock dynamics are affected. We do not include such effects in our calculations, treating the acceleration process as the test particle case. We do not include the effects of the shocks in the local conditions either, but one can consider them as averaged ones (for more precise calculations, properties of the shocks like velocity or compression ratio would be required, which is beyond present knowledge and it is absorbed by other parameters like $\eta$ and $\varrho$ ). $\xi$ might be interpreted as referring to a feedback effect on shock dynamics produced by accelerated particles.

The next constraint has been imposed:

$\frac{\mathrm{d} L_{\mathrm{dis}}(z)}{\mathrm{d} z} \geq \frac{\mathrm{d} L_{\mathrm{loss}}(z)}{\mathrm{d} z}$

${ }^{2}$ It depends on the angle between the magnetic field lines and the perpendicular direction to the shock surface, and on the shock speed in the plasma frame, as well as the diffusion coefficient in the shock region. $\eta$ is typically between $\sim 10^{-4}-10^{-1}$ (Protheroe 1999).

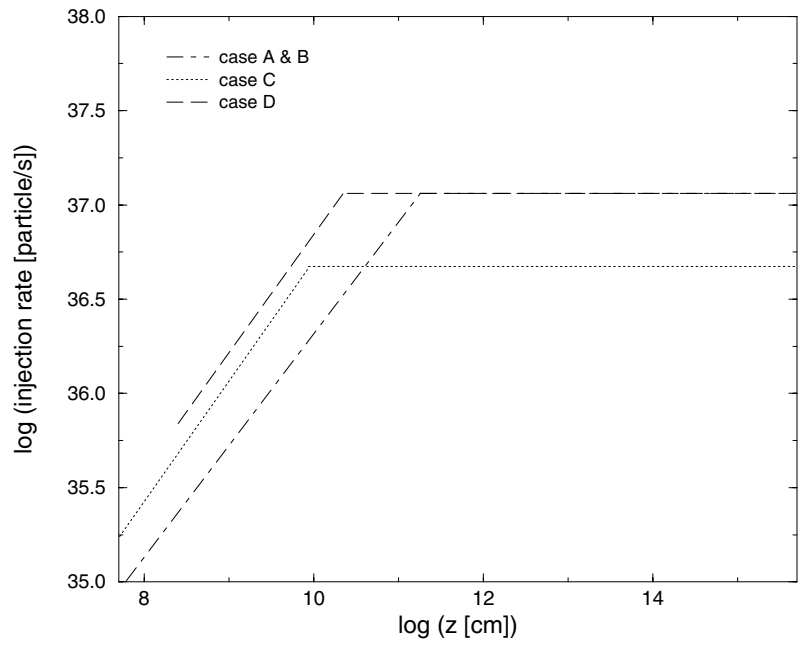

Fig. 3. The evolution of $\dot{Q}_{\text {inj }}$ along the jet for A-D. The free parameter values adopted for these cases are shown in Table 1.

where $\mathrm{d} L_{\text {loss }}(z) / \mathrm{d} z$ is the energy lost by length unit at different $z$ through radiative and adiabatic losses and has the form:

$\frac{\mathrm{d} L_{\mathrm{loss}}(z)}{\mathrm{d} z}=\int_{\gamma_{\min }}^{\gamma_{\max }(z)} \pi R_{\mathrm{jet}}^{2} n_{\mathrm{e}}(\gamma, z) \dot{\gamma}_{\mathrm{loss}} \mathrm{d} \gamma$,

provided that all the quantities are already in the compact object RF. $\mathrm{d} L_{\mathrm{dis}}(z) / \mathrm{d} z$ is the maximum dissipated luminosity per length unit, which is taken to evolve with $z$ like $\dot{\gamma}_{\text {gain }}$ :

$\frac{\mathrm{d} L_{\mathrm{dis}}(z)}{\mathrm{d} z} \sim \frac{C_{\mathrm{dis}}}{z}$

The normalization constant $C_{\mathrm{dis}}$ can be obtained by integrating the total amount of shock energy available per length unit along the jet, from $z_{0}$ to $z_{\max }$, and equating it to a suitable fraction $(\xi)$ of the jet kinetic luminosity:

$C_{\mathrm{dis}}=\frac{\xi L_{\mathrm{k} \text { esc }}}{\ln \left(z_{\max } / z_{0}\right)}$.

$\mathrm{d} L_{\mathrm{dis}}(z) / \mathrm{d} z$ has a weak $z_{\max }$-dependence. This quantity has been taken to be about $0.1 \mathrm{pc}$, where environmental effects on the jet properties could become significant, changing the characteristics of the jet, as it has been argued in Sect. 3.2.

Equating Eqs. (14) and (15) (through Eq. 8) gives $N_{\text {inj }}$ in the compact object RF, although this value cannot be higher than that derived from $\zeta$ either. In Fig. 3, we show the evolution of $\dot{Q}_{\text {inj }}$ for the four MQ cases studied here. Losses are strong enough well within the binary system to hold the total number of relativistic leptons injected per second (i.e. crossing a jet section at $z$ ) below the maximum allowed rate. As these particles are carried by the jet to larger $z$, losses are lower and the injected particle rate reaches its maximum value. For the cases considered here, synchrotron losses dominate over SSC, EC and relativistic Bremsstrahlung losses, although star IC losses can dominate at certain jet regions and/or for slightly smaller values of $B$. Therefore, the Klein-Nishina $(\mathrm{KN})$ effects are to be taken into account.

In Fig. 4, we see the evolution of the maximum energy of the relativistic particles with $z$. Radiative losses limit this maximum energy at spatial scales similar to the size of the binary 


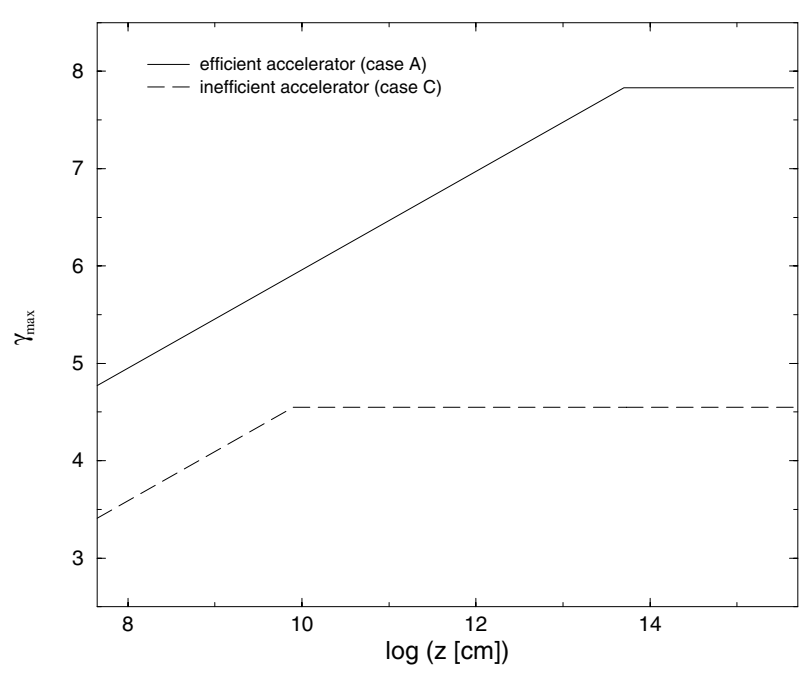

Fig. 4. The same as in Fig. 3 but for $\gamma_{\max }$ in the cases A and C.

system. At middle and large jet scales, the acceleration process is limited by the typical size of the jet and by the jet magnetic field. Both $z$-dependences get canceled, and the consequence is a constant value for $\gamma_{\max }$. We show the evolution of $\gamma_{\max }$ for two particularly interesting cases (Fig. 4): A and C. Since the amount of energy transferred to relativistic particles can be significant, we calculate the effects of this process on the jet Lorentz factor. Since different velocities in plasma motion generate shocks and the acceleration process, the jet Lorentz factor must be understood as an average value. The results for cases A-D are shown in Fig. 5. As is expected, the lower the acceleration efficiency (i.e. the transfer of energy from the bulk motion to the radiating particles), the lower the decrease in the bulk motion Lorentz factor, which goes down strongly at the base of the jet and is already stabilized at binary system scales. The radiative efficiency of the jets for our particular parameter choice (for $\varrho, \xi$ and $\eta$ ) is of about $1-10 \%$, not far from estimates obtained by different approaches (see, e.g., Fender 2001; Yuan et al. 2005). With the Lorentz factors given in Fig. 5, the initial jet kinetic luminosities are $8.7 \times 10^{36} \mathrm{erg} \mathrm{s}^{-1}, 8.7 \times$ $10^{36} \mathrm{erg} \mathrm{s}^{-1}, 3.5 \times 10^{36} \mathrm{erg} \mathrm{s}^{-1}$ and $4.9 \times 10^{37} \mathrm{erg} \mathrm{s}^{-1}$ for A-D respectively.

\section{The model: radiative processes in the jet}

\subsection{Radiation mechanisms}

We have accounted for synchrotron, relativistic Bremsstrahlung and IC emission (in both Thomson and $\mathrm{KN}$ regimes). Other leptonic radiative processes such as free-free transitions, thermal Bremsstrahlung, etc., have been neglected since jet emission is dominated by the non-thermal processes, although for a completely cold and powerful jet these processes would have observable effects. We show in Fig. 6 an example of the energy lost by leptons per volume and time units in the jet at different distances from the compact object. As is seen there, the dominant type of loss is synchrotron radiation, although the IC losses can be significant. Well outside the binary system, the dominant losses are the adiabatic ones. For certain ranges of the parameter

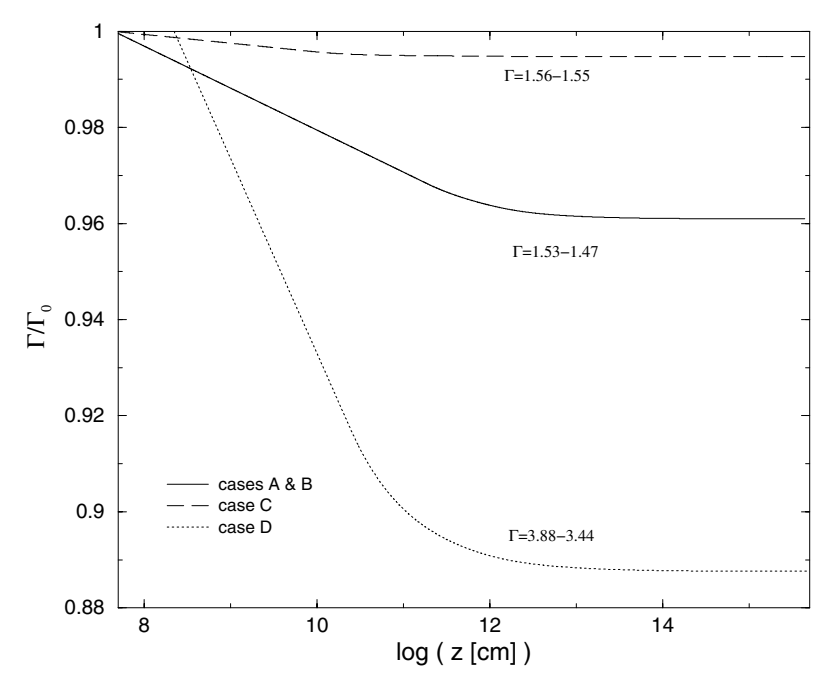

Fig. 5. The same as in Fig. 3, but for $\Gamma_{\text {jet }}$ normalized to its initial value in the four cases explored here.

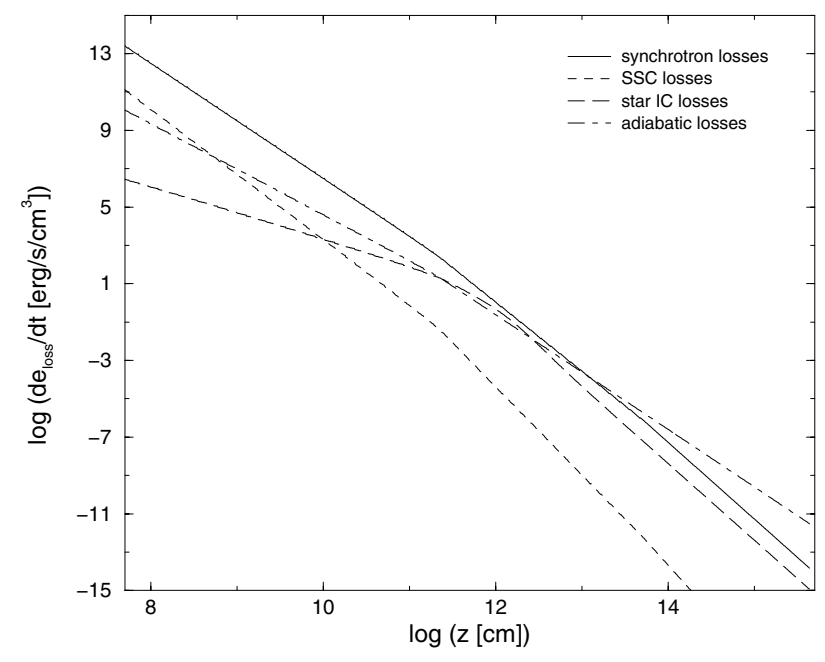

Fig. 6. The same as in Fig. 3 but for the energy per volume and time units lost by leptons, $\mathrm{d} e_{\text {loss }} / \mathrm{d} t$, now only for $\mathrm{A}$.

values, stellar IC (A-like but with low magnetic field) and corona IC (C) could dominate over synchrotron radiation. Relativistic Bremsstrahlung is negligible in general. Since the increase of the amount of relativistic particles stops when their number reaches a maximum value (recall $\zeta$ and Fig. 3), the decrease in the total emissivity becomes more serious at distances of about $10^{11} \mathrm{~cm}$ from the compact object.

Taking into account the physical conditions along the jet, we have computed the SEDs corresponding to the different mechanisms mentioned above. To calculate the spectrum of the radiation coming from the jet, we have used the energy distribution function of the relativistic leptons shown in Sect. 3.2, as well as the corresponding cross-sections of each process. For synchrotron emission, the magnetic field at different $z$ is given in Sect. 3.1, and we adopt the width of the jet as the length that determines whether synchrotron emission is either optically thin or optically thick. For external Bremsstrahlung, in the case of interaction with the stellar wind ions (the wind is considered as a completely ionized plasma), we take our calculations as 
an upper limit and assume that the target ion density is the wind density (i.e. that all the available wind particles diffuse within the jet); for internal Bremsstrahlung, the target ion density is the proton density of the jet, directly derived from $\dot{m}_{\text {jet }}$ since the jet is cold matter dominated. For EC emission, the target photon densities from the star, the disk and the corona are those described in Sect. 4.2, and in the case of internal Compton emission (basically, SSC), the target photon density has been calculated previously (i.e. synchrotron). All the $z$-dependences have been taken into account, dividing the jet in slices, each with homogeneous properties, and the overall jet emission has been integrated over all the slices. For further details concerning synchrotron, relativistic Bremsstrahlung and IC processes, as well as the electron energy loss expressions, we refer to the work by Blumenthal \& Gould (1970). The Doppler boosting effects in the observed spectra have been implemented as usual (e.g. Dermer \& Schlickeiser 2002).

\subsection{External photon fields}

The external seed photon sources considered in the model are the star, the disk and the corona. The star and the disk have been considered to be gray bodies, normalized to their total luminosities. The star photon distribution peaks at optical-UV energies, and the disk one peaks at $100 \mathrm{eV}$. This disk photon energy appears to be typical in the low-hard state when the optically thick disk is truncated far from the compact object and the disk matter does not reach temperatures as high as during more intense accretion states, when the inner disk radius shrinks. For the corona emission, we have assumed that it follows a powerlaw plus an exponential cut-off, peaking around $100 \mathrm{keV}$. Since IC interactions are studied first in the jet RF, we calculate the total energy densities and the spectral energy densities in this RF. Since expressions for the star, disk and corona photon energy density are originally in the compact object RF, they are transformed using a relationship found elsewhere (e.g. Dermer \& Schlickeiser 2002):

$U_{\epsilon_{0}, \Omega}(z)=\frac{U_{\epsilon_{0}^{\prime}, \Omega^{\prime}}^{\prime}\left(z^{\prime}\right)}{\Gamma_{\text {jet }}^{3}(1+\beta \mu)^{3}}$,

where $\Omega$ represents the photon direction, $\mu$ is $\cos \vartheta, \vartheta$ is the angle between the photon direction and the jet axis, and $\epsilon_{0}$ is the seed photon energy for IC interaction. The quantities with primes are in the compact object/observer RF, and in the jet RF otherwise. For the star photons we assume that they reach the jet, at a particular $z^{\prime}$, with the same direction, which depends on the orbital phase and $z^{\prime}$. In the case of disk photons, their direction is taken to be coming from behind the jet. To treat mono-directional seed photon fields, we have adopted the approach used by Dermer et al. (1992) ${ }^{3}$. Since the interaction angle between jet electrons at different $z^{\prime}$ and stellar photons affects the star IC emission and varies with the orbital phase, we recall the adopted criterion that the systems treated here are

\footnotetext{
${ }^{3}$ For the mildly relativistic jets treated here, the disk IC radiation coming from the counterjet is enhanced by the angular dependence of the IC interaction. However, for the adopted parameter values, this component is at most similar to the normal jet component.
}

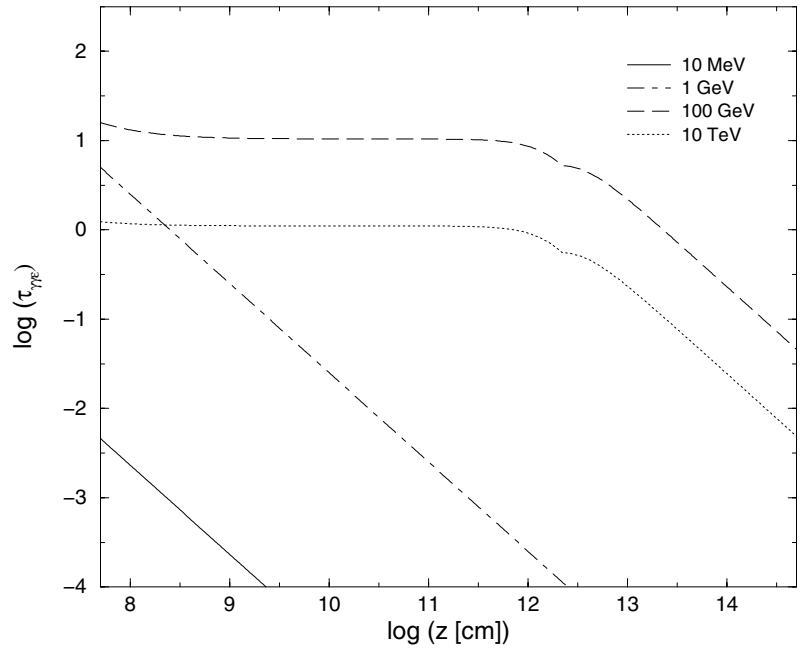

Fig. 7. Evolution of $\tau_{\gamma \gamma \epsilon^{\prime}}$ at different $z^{\prime}$. We have adopted the parameter values of the case A (see Table 1).

observed in such a way that at phase 0 or periastron passage, the compact object is in opposition to the observer. To apply the model to particular objects, the specific relationship between observer line of sight and compact object phase is needed. For corona photons, the jet base in this model is assumed to be located approximately in the external parts of this region. This source of photons is closer to the jet than the disk, and the interaction is significant only at the inner region of the jet due to the $1 / z^{2}$-decrease in density of corona photons. Thus, we have adopted the assumption that the corona field is roughly isotropic.

\subsection{Pair creation and annihilation within the jet}

We have investigated the effects of pair creation and annihilation phenomena on the jet emission by calculating the gamma-ray opacities $\left(\tau_{\gamma \gamma \epsilon^{\prime}}\right)$ for jet photons of different $\epsilon^{\prime}$ and produced at different $z^{\prime}$. To calculate opacities as well as the number of created and annihilated pairs, we have used the pair creation and annihilation rates given in Eqs. (3.7) and (4.6) of Coppi \& Blandford (1990). The evolution of the opacity is shown in Fig. 7. It is seen that opacities are higher in the vicinity of the compact object at energies between $1 \mathrm{GeV}-10 \mathrm{TeV}$. Gamma-ray absorption in the stellar UV photon field is significant for photon energies of $\sim 100 \mathrm{GeV}$ (for a deeper treatment of gamma-ray opacities due to the stellar photon field, see Romero et al. 2005; Böttcher \& Dermer 2005 and Dubus 2005). For certain parameter values, the opacity could be significant as well at $10 \mathrm{MeV}$ and a few $\mathrm{GeV}$ within the corona and disk fields (see also Romero et al. 2002).

The gamma-ray opacity by pair creation inside the jet is very high at $z^{\prime} \sim z_{0}^{\prime}$ because the target photon density is large and very sensitive to the jet width. If the jet width were larger, the internal opacity would be weaker, without affecting very much the overall production spectrum. Due to these uncertainties, the pair creation due to jet internal fields should be studied in a more detailed model in future work. 
Concerning annihilation rates inside this cold matter dominated jet, for any reasonable set of parameter values the luminosity that could be emitted in form of an annihilation line is too low to be distinguished from the continuum emission. Other models, like the one of Punsly et al. (2000), where a pure pair plasma is assumed, could produce detectable annihilation lines.

Observable predictions from considering pair creation phenomena in our model are presented and discussed briefly in Sects. 5 and 6, although we remark that the creation of pairs inside the jet could lead to the appearance of bumps due to the accumulation of particles at the energies of pair creation. To introduce such an effect properly requires a better knowledge of the particle injection function, which is beyond the scope of this work. Therefore, the high-energy gamma-ray band of the computed SEDs probably gives good enough flux estimates, although slopes could be slightly different as a result of all these subtle effects.

For those pairs that are created within the binary system, but outside the jet, the situation is different from that of pairs created inside. Starting with a determinate number of relativistic particles in the jet, plus the given jet conditions, one can consistently derive the SED of the produced radiation in the compact object RF. Thus, the spectrum is known, and it allows us to know precisely the number of absorbed photons and created pairs within the star, the disk and corona photon fields (for previous treatments of this, see Romero et al. 2002). From the previous result, it is possible to roughly estimate the radiation that is generated by those pairs through IC interaction with external source photons. Although it is a rough estimate, it is found to be in agreement with more detailed models of these processes (Khangulyan \& Aharonian 2005).

\section{Application of the model}

The different radiation components produced in the jet and the predicted SEDs have been computed for the four specific scenarios considered here. The effects of pair creation phenomena due to the external photon fields interacting with the produced gamma-ray photons are taken into account, and the secondary radiation produced by the created pairs is estimated. The calculations are performed at the periastron passage, when the compact object is in opposition to the observer and the interaction angle between star photons and jet leptons implies more luminosity for the star IC component (see Dermer et al. 1992), showing the importance of such an effect. However, such an angle depends on the electron energy, which should be taken into account in more detailed models of the IC interaction (e.g., Khangulyan \& Aharonian 2005).

The broad-band SEDs for cases A and B are presented in Figs. 8 and 9 respectively. The strong effects on the computed SED due to the presence of a massive star can be appreciated. The star IC component is very significant, partially because of the specific interaction angle between seed photons and leptons at phase 0 , and also because the interaction with stellar photons is more significant at large $z$, when the number of relativistic particles is higher (see Fig. 3), than for other photon fields. For $A$, gamma-gamma opacity is very high at VHE.

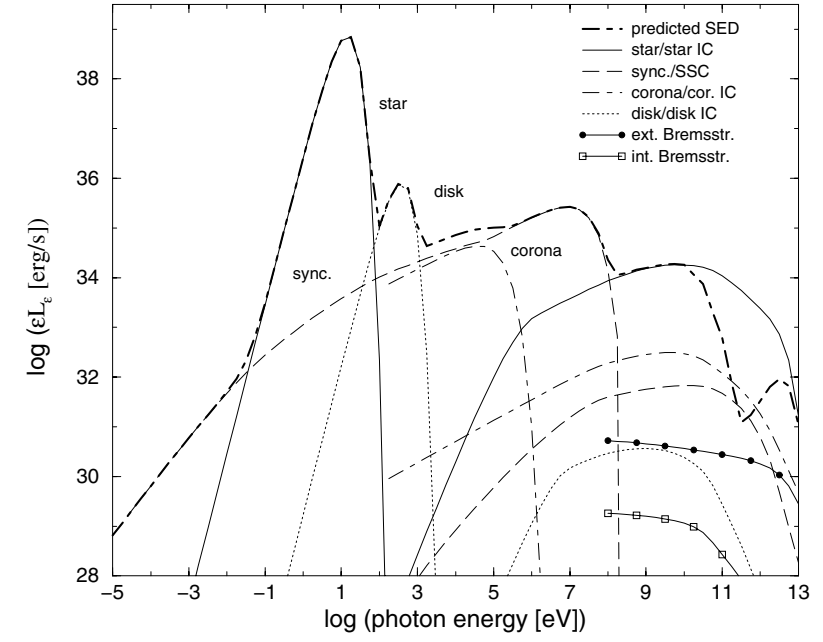

Fig. 8. Case A computed SED for the entire spectrum as it would be observed. Attenuation of the jet photons due to absorption in the external photon fields is taken into account, as well as the IC emission of the first generation of pairs created within them. Isotropic luminosity is assumed. The different IC, relativistic Bremsstrahlung, synchrotron and other seed photon fields are shown. For the several components, the production SED is shown. The corona photon field is also taken into account, but its effects on pair creation and subsequent emission are overcome by the synchrotron emission.

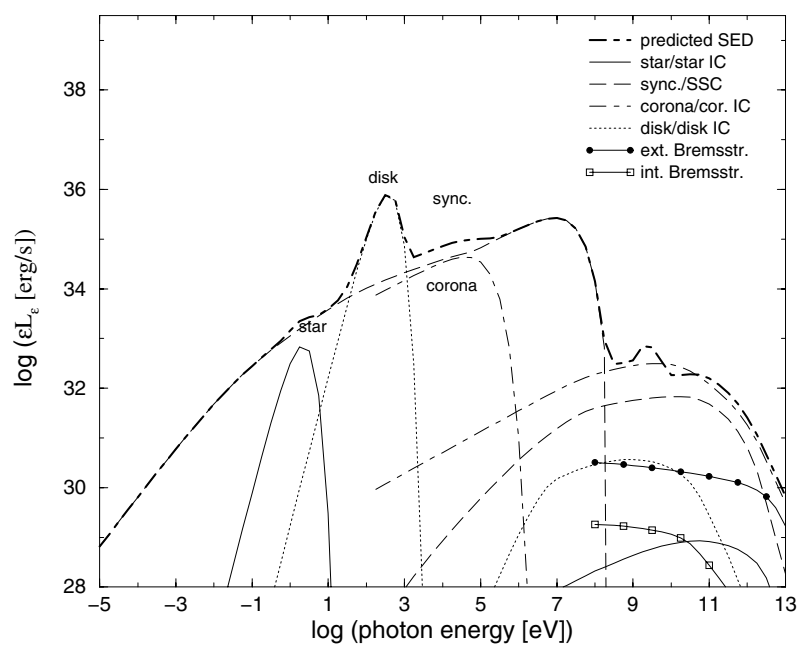

Fig. 9. The same as in Fig. 8 but for the case B. The small bumps present from beyond $100 \mathrm{MeV}$ come from the IC radiation emitted by those leptons generated by pair creation in the disk and the stellar photon field. These pair components are not made explicit in the plot for clarity.

We recall that the disk and corona emission have been assumed to radiate just a few per cent of the accretion power. As accretion does not dissipate a significant fraction of the available energy via either disk or corona radiation, the jet can carry more energy and matter for the same ejection velocity (and the assumptions put forward in Sect. 3.1 are valid). The acceleration efficiency has been assumed to be high.

In Fig. 10, the broad-band SED of case $C$ is shown. We have increased the disk and the corona emission, fixing the jet velocity. This implies a lighter jet than in the two previous cases. Also, we have modified the acceleration efficiency of the jet 


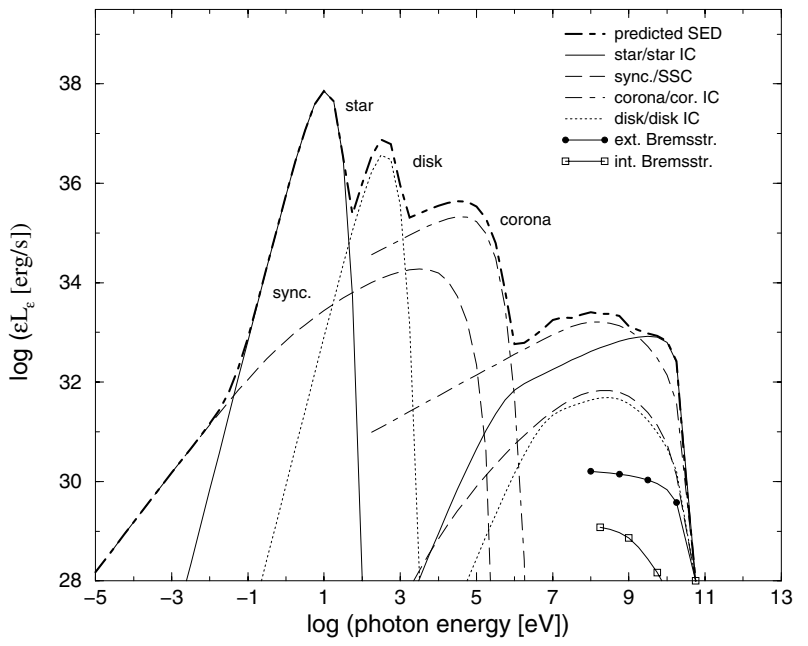

Fig. 10. The same as in Fig. 8 but for the case $C$ with a jet with low acceleration efficiency. For this particular situation it is possible to see the small bump at $10 \mathrm{MeV}$ produced through IC scattering by the pairs created in the corona photon field.

for this particular case to a smaller value than those previously used. The system harbors a massive star, although neither as massive nor as bright as in A. In the case of D, whose SED is plotted in Fig. 11, the matter content of the jet has been reduced to a smaller value, fixing again disk and corona emission and particle acceleration efficiency as in A and B. This implies that the jet motion will be more relativistic than in previous cases. This is unusual in what has been said in this work. Our purpose in studying this particular scenario is to show that an ultra luminous X-ray source can be reproduced through strong beaming and small viewing angles. Since the goal in this specific scenario was to obtain very high $\mathrm{X}$-ray fluxes, the compact object has been considered a $15 M_{\odot}$ black hole, allowing for higher accretion rates since the Eddington limit is higher for more massive accreting objects. We have also increased the shock maximum energy dissipation efficiency up to $\xi=0.5$.

The SEDs obtained at high energies resemble roughly those obtained applying the model presented in Bosch-Ramon et al. (2005a). This is because the IC emission dominates at high energies and, at least in some of the cases, the dominant IC component is the same as in that model. However, the physical motivation of the present work goes much further than before, with predictions concerning radio, variability and jet physical properties that could not be provided in the previous model.

\subsection{Spectral properties}

At gamma-rays, a different component is the main one in each explored scenario. For A, the star Comptonized photons are dominant, reaching $10^{34} \mathrm{erg} \mathrm{s}^{-1}$ at $100 \mathrm{MeV}$, with a photon index of about 2 , and $5 \times 10^{32} \mathrm{erg} \mathrm{s}^{-1}$ at $100 \mathrm{GeV}$. At VHE, due to the gamma-gamma absorption, the model predicts a soft photon index that hardens at higher energies. There could be additional spectral features related to the secondaries created in the corona, the disk and the star photon field. These features would appear as bumps at energies of a few $\mathrm{GeV}$ for disk pairs and a few tens of $\mathrm{GeV}$ for star photons (negligigle in $A$ ). Even

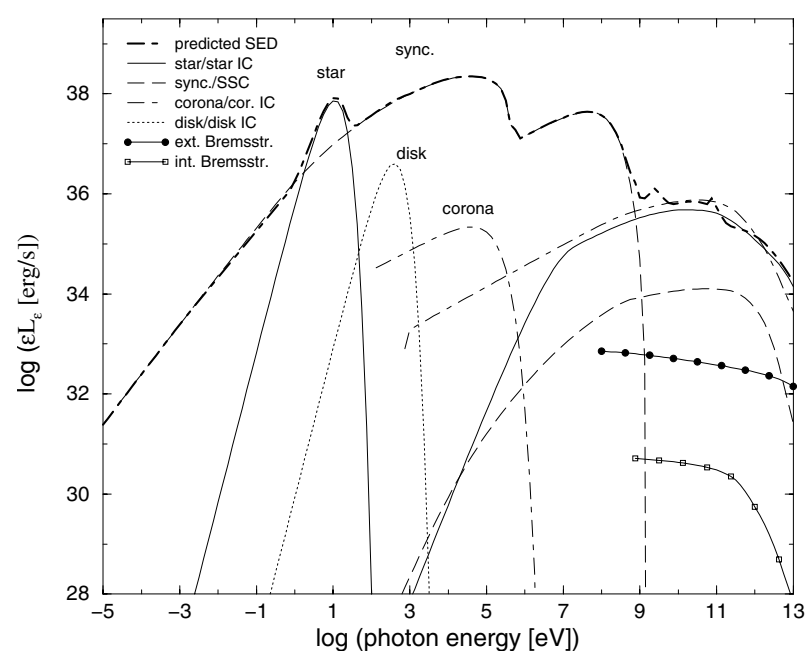

Fig. 11. The same as in Fig. 8 but for the case D.

for negligible disk and corona sources, a decrease in the predicted flux beyond $50 \mathrm{GeV}$ is unavoidable if it is a relatively close and massive binary system. In the case of $\mathrm{B}$, the dominant components are the corona IC and the SSC ones, with luminosities of about $10^{33} \mathrm{erg} \mathrm{s}^{-1}$ at $100 \mathrm{MeV}$ with a photon index of 2 (beyond the synchrotron component, see Fig. 9), and few $10^{32} \mathrm{erg} \mathrm{s}^{-1}$ at $100 \mathrm{GeV}$. In this case, however, the low mass star photon field does not significantly affect the VHE spectrum, and only a small bump due to the secondaries created within the disk photon field is visible in the SED. Therefore, the photon index beyond $10 \mathrm{GeV}$ gets softer because of the $\mathrm{KN}$ effect, although it is a smoother steepening than in $\mathrm{A}$. Concerning $\mathrm{C}$, the luminosity dominated by the corona IC emission at $100 \mathrm{MeV}$ is about $10^{33} \mathrm{erg} \mathrm{s}^{-1}$ and the photon index is similar to the one predicted in the previous cases. Otherwise, only disk and corona gamma-gamma absorption effects are significant, visible in the plots at about $1 \mathrm{GeV}$ and $10 \mathrm{MeV}$ respectively (see Fig. 10). The star IC component turns to dominate beyond a few GeV. For D, the Doppler boosting affects synchrotron radiation, which reaches $100 \mathrm{MeV}$ with luminosities higher than $10^{37} \mathrm{erg} \mathrm{s}^{-1}$. However, the SSC component is not dominant at all because it is very sensitive to the jet density, and the jet now is the least dense among the four studied cases. Therefore, beyond $1 \mathrm{GeV}$ the source is dominated by the corona and star IC components, with a photon index softer than 2 and some absorption produced by the star photon field. Small peaks of the IC emission of the secondaries are also present produced within the star and the disk photon fields.

In X-rays, the emission is synchrotron dominated for most cases (except for $\mathrm{C}$ ). This is because the magnetic field is below but not far from equipartition. The matter energy density at the jet base is so high that the magnetic field reaches values around $10^{5} \mathrm{G}$, allowing synchrotron radiation to dominate up to soft gamma-ray energies. Similar results have been obtained by Markoff et al. $(2001,2003)$ for the case of shock acceleration limited mainly by synchrotron losses. In our model particle acceleration is limited by shock energy dissipation efficiency, jet size, adiabatic and all the radiative losses (including $\mathrm{KN}$ regime for IC losses). Because of the evolution 
of $\gamma_{\max }$ that rises when $z$ is larger (for reasonable parameter values), the synchrotron spectrum changes smoothly at energies around $100 \mathrm{keV}$. Nevertheless, the disk and corona are not negligible in general, and in the case of $\mathrm{C}$, disk and corona overcome the jet radiation up to $1 \mathrm{MeV}$. D as an X-ray source is extremely bright. This result shows that a light and fast jet observed from very small viewing angles might turn out to be an ULX. Because of the low probability of being observed, it is more likely to detect them in other galaxies (for previous works on MQs as ULXs, see, e.g., Georganopoulos et al. 2002; Körding et al. 2002).

Below stellar emission energies, synchrotron radiation dominates again. If stellar emission were reprocessed by absorption and shifted to lower energies, it is likely that the far infrared would still be dominated by the enshrouded stellar component. At radio frequencies, there is significant radio emission, with isotropic luminosities of about $10^{29} \mathrm{erg} \mathrm{s}^{-1}$ at $5 \mathrm{GHz}$ (few mJy at $3 \mathrm{kpc}$ ). Our model predicts core-dominated emission, strongly self-absorbed due to the high efficiency of the inner jet to radiate through synchrotron process. The SED in the radio band corresponds to a spectral index equal to zero (or $\epsilon L_{\epsilon} \propto \epsilon^{+1}$ ), as it would be expected from a conical jet. $\mathrm{C}$ presents the weakest radio emitting jet, weaker than in $\mathrm{A}$ and $\mathrm{B}$, and $\mathrm{D}$ is the strongest radio source due to the Doppler boosting. Further aspects on radio emission in our scenario are commented in next section.

\subsection{Variability}

Variability through changes in the stellar mass-loss density profile is introduced in the model in a consistent way when referring to high-mass microquasars. In Fig. 12, the SEDs of $\mathrm{A}$ at phases 0.3 (accretion maximum), 0 (periastron passage) and 0.7 (accretion minimum) are shown. For clarity, we have split the overall spectrum in three bands: radio, X-rays and gamma-rays. Emission varies due to orbital eccentricity for a spherical slow wind accreting system with constant wind velocity. Even for the simple model considered here, it is possible to see from the figures the complex evolution of radiation. At radio and X-ray frequencies, the different fluxes associated with different phases are correlated with the accretion rate. Since the velocity of the compact object relative to its surrounding medium changes along the orbit, the periastron is not directly associated with the highest flux. However, at gamma-rays, the particular angle of interaction between star photons and jet electrons implies that the stellar IC is dominant, this phase being associated with a peak in gamma-ray emission. More complex variations in the accretion, affecting jet ejection and radiation, could be introduced through the function reproducing the stellar mass-loss density profile. Other timescales of variability, linked to disk phenomena, could be introduced through the parameter that controls the amount of matter that goes to the jet, although it is beyond the scope of this work. Also, the radio variability pattern depends on the scale of the radio emitting region, which is the inner jet for the parameter values adopted here.
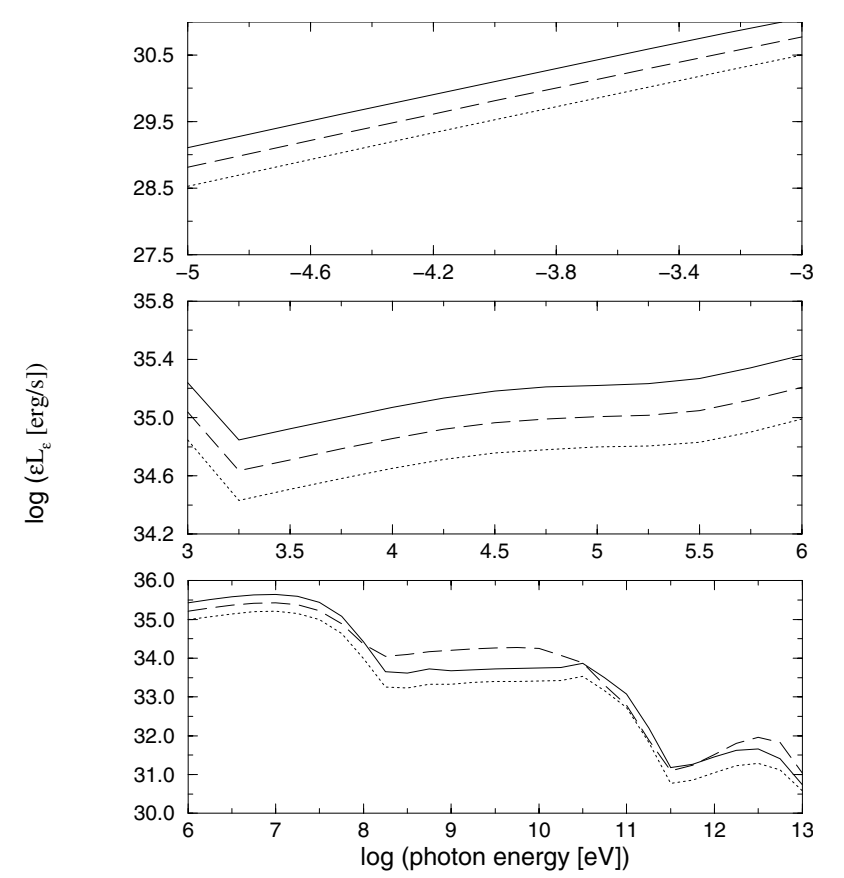

Fig. 12. The case A, three predicted SEDs at radio, X-ray and gamma-ray energy bands, corresponding to three different orbital phases: 0 (periastron passage, dashed line), 0.3 (highest accretion rate, solid line), and 0.7 (smallest accretion rate, dotted line).

\section{Discussion}

We conclude that persistent jets in MQs in the low-hard state, despite accreting at relatively low rate, and under reasonable conditions for the jet matter, energy and magnetic field, can radiate with significant luminosities from radio to gamma-rays. The model provides predictions about the shape of the SEDs and points to MQs as VHE sources, as has been recently confirmed by Aharonian et al. (2005) in the case of LS 5039. It also predicts variability at different ranges of energy. The importance of the synchrotron cooling channel for relativistic particles in the jet is high. This points to the fact that, for certain objects, the jet could overcome at all wavelengths any other emitting region of the MQ, except the star itself for high mass systems. We note that for a relatively weak disk and corona $\left(10^{34} \mathrm{erg} \mathrm{s}^{-1}\right)$, and even with $\varrho \sim 0.01$ (one tenth of the one adopted here), the spectrum would be jet dominated and well described by a power-law at X-ray wavelegnths (see also Paredes et al. 2005). An observational feature that could determine whether X-rays come from a jet is the dependence of X-ray fluxes on the accretion rate, if the latter can be estimated. In the scenario presented here, X-rays vary with $\dot{m}_{\text {acc }}$, but it is also indirectly connected to $B$ and $\gamma_{\max }$, the latter also being sensitive to the overall jet conditions and size, and both quantities depend on the accretion rate as well. Long exposure multiwavelength observations will be required if a relationship between radiation components of different origin is to be found with high accuracy, particularly considering the moderate X-ray fluxes of permanent jet sources like LS 5039, which do not appear to follow the typical behavior of X-ray binaries in the low-hard state (Bosch-Ramon et al. 2005c). An additional observational feature that would favor the jet as the origin of 
the X-rays would be the detection of some amount of polarization in this radiation.

At gamma-rays, our jet model, more detailed and in accordance to the current knowledge of MQs than our previous works, still shows that MQs could be behind some EGRET sources (likely those variable and located in the galactic plane), and also predicts a different evolution of emission at different energies due to IC angular dependence interaction, some strange features like radiation bumps and depressions in the spectra due to gamma-gamma absorption with external photon fields, and a non trivial relationship between pair creation and particle injection within the jet itself. GLAST and AGILE, with a sensitivity at least several times better than that of EGRET, should be able to detect microquasars even when they are low mass systems (case B) and/or they have weaker jets (case C). The detection of the microquasar LS 5039 by HESS shows that the efficiency of the particle acceleration processes should be high. Due to the strong photon absorption beyond $\sim 100 \mathrm{GeV}$, it might be that the bulk of the TeV emission came from regions where stellar photon density is not significant.

Although we are using accretion rates which are a small fraction of the Eddington luminosity, the wind velocity is required to be low to power accretion and the jet itself. This points to the fact that $\mathrm{O}$ stars with spherical fast winds would not be able in general to power some of the compact jets observed in the galaxy, implying that some special wind density profile should be given, likely produced by the presence of the compact object plus other factors like companion star rotation, etc. (Paredes et al. 2005). For the launching radius, we have taken $4 R_{\text {Sch }}$ to reach mildly jet Lorentz factors. Although our approach to estimate the energy balance between the jet and accretion is rough, this value for $r_{1}$ is between the last stable orbit and the limits of the corona-like region, consistent with the present state of knowledge on this issue.

\subsection{Radio emission}

Previous models for leptonic emission from a magnetized compact jet predicted core dominated radio emission as observed in several galactic and extragalactic sources (for XRBs jet models, see, e.g. Markoff et al. 2001; for extragalactic jet models, see, e.g. Ghisellini et al. 1985). Our results are similar to those presented in these previous works, where radio emission that comes from the inner jet regions is strongly self-absorbed, becoming optically thin further down the jet. On the other hand, it is difficult to correctly model jet radio emission in some high-mass XRBs, like that presented by LS 5039 (Martí et al. 1998; Ribó et al. 1999). A deeper discussion on this subject is presented in Paredes et al. (2005). We advance however that a $z$-dependence for the parameter $\eta$, or also a less stringent restriction of $\zeta$, could lead to a higher production of radio emission in optically thin regions of the jet.

A correlation is seen between the luminosity in the radio band and that at X-rays (Corbel et al. 2003; Gallo et al. 2003) that appears to be present in different sources with very different accretion rates and compact object mass values
(Falcke et al. 2004). In the case of our model, when the source is jet dominated at X-ray wavelengths (cases A, B and D), the correlation is fulfilled. Otherwise, for corona-dominated sources (case C) the correlation cannot be reproduced, since we are not modeling the corona. Instead, we adopt a typical spectrum and a certain luminosity in each case.

\section{Summary}

We have developed a detailed leptonic model for an MQ jet that can reproduce the emission observed from radio to gamma-ray energies, and makes precise predictions for very high energies. The basic assumptions of the model are a cold-matter dominated jet, with a magnetic field close to but below equipartition that is entangled with and dynamically dominated by jet cold matter. With the knowledge of the system parameters, given a simple stellar mass-loss density profile, and varying the jet to advected matter ratio and the acceleration efficiency, a set of broad-band SEDs has been computed. Also, the opacity due to photon-photon interactions was taken into account to calculate the predicted SEDs. The absorption can significantly distort the production spectrum beyond $10 \mathrm{GeV}$ mainly due to the effect of stellar photons in the case of massive companion stars. The opacity can be important even at lower energies when disk and corona radiation densities are high enough. The model shows that pair creation inside the jet could affect jet radiation. This aspect will be investigated accurately in future work. For systems where orbital eccentricity or other stellar mass-loss asymmetries are present, consistent predictions of the variability emission pattern of the source can be obtained.

New generation gamma-ray instruments, both satelliteborne or ground-based, like GLAST, AGILE, MAGIC or HESS can be used to test and constraint the high-energy predictions and assumptions of the model.

Acknowledgements. We thank an anonynous referee for useful comments and suggestions that significantly improved the manuscript. We are grateful to Evgeny Derishev, Dmitri Khangulyan, Felix Aharonian and John Kirk for useful discussions on particle acceleration and plasma physics. V.B-R. and J.M.P. acknowledge partial support by DGI of the Ministerio de Educación y Ciencia (Spain) under grant AYA-2004-07171-C02-01, as well as additional support from the European Regional Development Fund (ERDF/FEDER). During this work, V.B-R has been supported by the DGI of the Ministerio de (Spain) under the fellowship BES-2002-2699. G.E.R is supported by the Argentine Agencies CONICET and ANPCyT (PICT 03-13291). This research has benefited from a cooperation agreement supported by Fundación Antorchas.

\section{References}

Aharonian, F., Akhperjanian, A. G., Aye, K. M., et al. 2005, Science, 309, 746

Atoyan, A. M., \& Aharonian, F. A. 1999, MNRAS, 302, 253

Bell, A. R. 1978, MNRAS, 182, 443

Biermann, P. L., \& Strittmatter, P. A. 1987, ApJ, 322, 643

Blumenthal, G. R., \& Gould, R. J. 1970, Rev. Mod. Phys., 42, 237

Böttcher, M., \& Dermer, C. D. 2005, ApJ, 634, L81

Bondi, H. 1952, MNRAS, 112, 195

Bosch-Ramon, V., \& Paredes, J. M. 2004, A\&A, 417, 1075 
Bosch-Ramon, V., Romero, G. E., \& Paredes, J. M. 2005a, A\&A, 429, 267

Bosch-Ramon, V., Aharonian, F. A., \& Paredes, J. M. 2005b, A\&A, 432, 609

Bosch-Ramon, V., Paredes, J. M., Ribó, et al. 2005c, ApJ, 628, 388

Coppi, P., \& Blandford, R. D. 1990, MNRAS, 245, 453

Corbel, S., Fender, R. P., Tzioumis, A. K., et al. 2002, Science, 298, 196

Corbel, S., Nowak, M. A., Fender, R. P., Tzioumis, A. K., \& Markoff, S. 2005, ApJ, 400, 1007

Corbel, S., Kaaret, P., Fender, R. P., et al. 2005, ApJ, 632, 504

Dermer, C. D., \& Schlickeiser, R. 2002, ApJ, 575, 667

Dermer, C. D., Schlickeiser, R., \& Mastichiadis, A. 1992, A\&A, 256, L27

Dubus, G. 2005, A\&A, submitted [arXiv:astro-ph/0509633]

Esin, A. A., McClintock, J. E., \& Narayan, R. 1997, ApJ, 489, 865

Falcke, H., Körding, E., \& Markoff, S. 2004, A\&A, 414, 895

Fender, R. P. 2001, MNRAS, 322, 31

Fender, R. P., Gallo, E., \& Jonker, P. G. 2003a, MNRAS, 343, L99

Fender, R. P., Migliari, S., \& Méndez, M. 2003b, NewAR, 47, 481

Fukue, J., Tojyo, M., \& Hirai, Y. 2001, PASJ, 53, 555

Gallo, E., Fender, R. P., \& Pooley, G. G. 2003, MNRAS, 344, 60

Georganopoulos, M., Aharonian, F. A., \& Kirk, J. G. 2002, A\&A, 388, L25

Ghisellini, G., Maraschi, L., \& Treves, A. 1985, A\&A, 146, 204

Grenier, I., Kaufman Bernadó, M. M., \& Romero, G. E. 2005, Ap\&SS, 297, 109

Hartman, R. C., Bertsch, D. L., Bloom, S. D., et al. 1999, ApJS, 123, 79

Heinz, S. 2002, A\&A, 388, L40

Hjellming, R. M., \& Johnston, K. J. 1981, ApJ, 246, L141

Hujeirat, A. 2004, A\&A, 416, 423

Kaufman Bernadó, M. M., Romero, G. E., \& Mirabel, I. F. 2002, A\&A, 385, L10

Khangulyan, D., \& Aharonian, F. A. 2005, in preparation

Körding, E., Falcke, H., \& Markoff, S. 2002, A\&A, 383, L13

Markoff, S., Falcke, H., \& Fender, R. 2001, A\&A, 372, L25

Markoff, S., Nowak, M., Corbel, S., et al. 2003, A\&A, 397, 645

Marshall, H. L., Canizares, C. R., \& Schulz, N. S. 2002, ApJ, 564, 941

Martí, J., Paredes, J. M., \& Ribó, M. 1998, A\&A, 338, L71

Massi, M., Ribó, M., Paredes, J. M, et al. 2004, A\&A, 414, L1
Mastichiadis, A., \& Kirk, J. G. 1995, A\&A, 295, 613

McClintock, J. E., \& Remillard, R. A. 2004, to appear as Chapter 4 in Compact Stellar X-ray Sources, ed. W. H. G. Lewin, \& M. van der Klis (Cambridge University Press) [arXiv: astro-ph/0306213]

Meier, D. L. 2003, New Astron. Rev., 47, 667

Migliari, S., Fender, R., \& Méndez 2002, Science, 297, 1673

Mirabel, I. F., Rodriguez, L. F., Cordier, B., Paul, J., \& Lebrun, F. 1992, Nature, 358, 215

Mirabel, I. F., Dhawan, V., Chaty, S., et al. 1998, A\&A, 330, L9

Mirabel, I. F., \& Rodríguez, L. F. 1999, ARA\&A, 37, 409

Paredes, J. M., Martí, J., Ribó, M., \& Massi, M. 2000, Science, 288, 2340

Paredes, J. M., Ribó, M., Ros, E., et al. 2002, A\&A, 393, L99

Paredes, J. M., Bosch-Ramon, V., \& Romero, G. E. 2005, A\&A, submitted [arXiv: astro-ph/0509095]

Protheroe, R. J. 1999, in Topics in Cosmic-Ray Astrophysics; Horizons in World Physics, ed. M. A. DuVernois (Commack, New York: Published by Nova Science Publishers, Inc.), 230, 247

Punsly, B., Romero, G. E., Torres, D. F., \& Combi, J. A. 2000, A\&A, 364,552

Rees, M. J. 1978, MNRAS, 184, 61

Reig, P., Ribó, M., Paredes, J. M., \& Martí, J. 2003, A\&A, 405, 285

Ribó, M., Paredes, J. M., \& Martí, J. 1999, NewA Rev., 43, 545

Romero, G. E., \& Orellana, M. 2005, A\&A, 439, 237

Romero, G. E., Kaufman Bernadó, M. M., \& Mirabel, I. F. 2002, A\&A, 393, L61

Romero, G. E., Torres, D. F., Kaufman Bernadó, M. M., \& Mirabel, I. F. 2003, A\&A, 410, L1

Romero, G. E., Grenier, I. A., Kaufman Bernadó, M. M., Mirabel, I. F., \& Torres, D. F. 2004, ESA-SP, 552, 703

Romero, G. E., Christiansen, H., \& Orellana, M. 2005, ApJ, 632, 1093

Shakura, N. I., \& Sunyaev, R. A. 1973, A\&A, 24, 337

Sikora, M., Begelman, M. C., Madejski, G. M., \& Lasota, J. 2005 , ApJ, 625, 72

Spada, M., Ghisellini, G., Lazzati, D., \& Celotti, A. 2001, MNRAS, 325,1559

Spencer, R. E. 1979, Nature, 282, 483

Torres, D. F., Romero, G. E., \& Mirabel, F. 2005, ChJAA, 5, 183

Yuan, F., Cui, W., \& Narayan, R. 2005, ApJ, 620, 905 